\title{
EDUCATIONAL
} TECHNOLOGY

\section{The Luria model of information processing}

\author{
John Angus \\ Lecturer, Institute of Early Childhood Studies \\ Newtown Campus
}

\section{Introduction}

The concept of individual differences is as old as the study of Psychology. In recent years, educators have recognised that the differing abilities of learners to process information has important implications for learning outcomes. The study of the interaction between the characteristics of a given learner and his or her success under a given treatment has become known as Aptitude Treatment Interaction ATI (Cronbach and Snow, 1977). Salamon (1972), has described three heuristic models: the Remedial Model; the Compensatory Model; and the Preferential Model, all of which have the worthy objective of helping individual learners to achieve the fullest potential of which they are capable in the given area.

ATI has held out great promise to instructional designers and to educational technologists, but the promise has been largely unfulfilled. In the media field one of the exceptions to this generalisation is the work undertaken by Salamon in his studies of the codes of film and television (Salamon 1977, 1979). One of the reasons for this relative lack of success in adapting instruction to individual needs, has been the difficulty experienced in identifying significant learner aptitudes.

A model of individual difference which holds considerable potential for the educational technologist is one developed from the research findings of A R Luria (1966). Luria's clinical investigations of brain damaged patients led him to propose that the brain processes information in two fundamentally different modes, which he describes as simultaneous integration and successive integration. (Luria, 1966). Simultaneous integration is concerned with the perception and processing of stimuli which are instantaneously surveyable. The ability to visualise and to mentally manipulate images is closely associated with this processing 
mode. Successive integration, by comparison, is concerned with the linking together in a chain like way a series of stimuli which arrive one after the other. The recall of a series of digits, such as a telephone number or the smooth flow of words in speech are dependent upon this chaining or successive integration.

Over the last few years a number of researchers have been seeking to operationalise the neurological model of Luria and to apply the insights so gained to practical learning situations. There have been two principal groups of workers researching in their area, one group originating in North America (Des, 1973, Kirby, 1978) and a second group from the University of New England (Fitzgerald 1973; Green 1977; Ransley 1981; Walton 1983; etc).

The two groups of researchers have independently developed tests which have sought to model psychometrically, the simultaneous and sequential processing of Luria. The test batteries resulting have been factor analysed and have consistently demonstrated the presence of factors which could be labelled "simultaneous" and "successive". In addition to these two elemental processing factors the New England group has included a number of tests in their batteries designed to measure, selective attention, in order to better model Luria's later writings. The tests which have been used in these factor analytic studies have been accepted on their face validity with the Luria clinical tasks. The study described in this paper set out to examine the validity of the psychometric model against the clinical tasks used by Luria.

\section{The validity of the psychometric model}

It is not entirely surprising when a battery of 10 or 12 tests, which includes, measures purporting to assess simultaneous processing, successive processing and selective attention produce factors which can be labelled with these terms. A question of considerable importance, however, is the extent to which the factors so labelled correspond with the original and neurologically significant concepts. In order to examine the validity of the psychometric model developed from Luria's theory against Luria's clinical tasks a study was undertaken using 320 grade 2 children from the Sydney area.

The children of the sample completed a battery of 10 tests which included tests of simultaneous processing figure copying of given and inverted figures, tests of successive processing recall of digits and letter strings, and tests of selective attention recalling letters or digits from a mixed letter and number string and two Cloze procedure reading comprehension tests. The successive tests were marked so as to emphasise the recall of letters or digits in the correct sequence. 
A factor analysis of the scores resulted in the emergence of the three anticipated factors simultaneous processing, successive processing, and selective attention. An examination of the three factor structure in comparison with a four factor structure showed that the four factor structure was more easily interpreted and better accommodated the data. The fourth factor, which had significant loadings from the two reading tests, was labelled reading/verbal fluency. On the basis of this analysis, the children of the sample were identified as high, medium or low on each of the four factors. To maximise the contrasts between children of differing aptitudes only children in the extreme groups were used in the study, that is, children of high aptitude and of low aptitude on each factor. Since four factors were being considered and two levels for each factor, the number of cells was $2 \times 2 \times 2 \times 2$. Four children were randomly selected from each of these sixteen cells to undertake a clinical interview, based on Luria's clinical studies.

\section{Criteria for the selection of tasks for the clinical interview}

It should be noted that the purpose of the present study involving Luria's clinical tasks was quite different from that of Luria; the real interest in the present study lay in the significant individual differences which had been identified by the base line tests. These considerations suggested that some of Luria's tasks may not be relevant. In sampling Luria's tasks two criteria were used:

i. the tests should have face validity with one of the four factors identified in Study 1.

ii. the test should have some relevance to audiovisual instruction in the classroom.

The first criterion was important with respect to examining the validity of the psychometric model and the second criterion was important in linking the psychometric study to practical instruction.

The application of these two criteria led to the identification of 34 tests which could be administered in one session. Table 1 presents an outline summary of Luria's Neurological Investigations and Table 2, lists the clinical tasks selected, and the factors of the base line study which were predicted as correlating positively with the tests.

\section{Collection of data}

Six teacher-education students were trained as interviewers for the clinical study. The students undertook the interviews as a formal course requirement for a college course "Communication and Media". The protocol for the interview was explained to the students and a training 
tape was used to develop consistency between interviewers. The training tape consisted of a number of previously recorded interviews with children. The interviewers listened to the tape and scored each item. Difficulties arising were discussed and the training process was continued until the interviewers felt confident and their judgements were consistent. A simple rating scale, following Luria's method was used - children were rated as having no difficulty, slight difficulty, or severe difficulty with each task.

The children selected for interview were randomly assigned to the interviewers but with a degree of stratification so that each interviewer saw a range of abilities within the four factors. The interviewers were "blind" with respect to the ability levels of the children on the factors to avoid bias.

\section{Analysis of the data}

The product-moment correlations between each of the variables in the tests and the original factor scores derived from the base line was computed. This analysis is presented in Table 3.

Table 1: Summary of Luria's Neuropsychological Investigation

\begin{tabular}{|c|c|}
\hline A. & $\begin{array}{l}\text { Purpose and procedure of the neuropsychological } \\
\text { investigation }\end{array}$ \\
\hline \multirow[t]{5}{*}{ B. } & Preliminary conversation \\
\hline & State of Consciousness \\
\hline & Premorbid Level \\
\hline & Attitude \\
\hline & Complaints \\
\hline C. & Cerebral Dominance \\
\hline \multirow[t]{4}{*}{ D. } & Motor Functions \\
\hline & Hands \\
\hline & Oral Praxis \\
\hline & Speech Regulation \\
\hline \multirow[t]{2}{*}{ E. } & Acoustico-Motor Organisation \\
\hline & Perception and reproduction of pitch \\
\hline F. & Higher Cutaneous and Kinaesthetic Functions \\
\hline G. & Higher Visual Functions \\
\hline H. & Impressive Speech \\
\hline J. & Expressive Speech \\
\hline K. & Writing and Reading \\
\hline L. & Arithmetical Skill \\
\hline M. & Mnestic Processes \\
\hline N. & Intellectual Processes \\
\hline
\end{tabular}

(Anne-Lise Christensen, 1974) 
Within each of these areas of investigation, Luria used a number of diagnostic tests from which a selection of tasks was made for the present study.

Table 2: The Tasks Selected for Clinical Interviews and Positive Correlations Between Luria's Clinical Task and Base Line Factors

\begin{tabular}{|c|c|c|c|c|c|}
\hline \multirow{2}{*}{\multicolumn{2}{|c|}{ TEST }} & \multicolumn{4}{|c|}{ Predicted to correlate with } \\
\hline & & I & II & II & IV \\
\hline 1 & Handedness & & & & \\
\hline 2 & Verbal Hemisphere & & & $x$ & \\
\hline 3 & Motor Hand Function & $x$ & $x$ & & \\
\hline 4 & Rhythmic Structures & $x$ & & & $x$ \\
\hline 5 & Visual Functions & & & $x$ & \\
\hline 6 & Reading Fluency & & & $x$ & $\mathrm{x}$ \\
\hline 7 & Simple Pictures & & $x$ & & \\
\hline 8 & Complex Pictures & & $x$ & & \\
\hline 9 & Silhouette Photo & & $x$ & & \\
\hline 10 & Overlapping Pictures & $x$ & $x$ & & \\
\hline 11 & Chessboard Figures & & $x$ & & \\
\hline 12 & Patterns & $x$ & $x$ & & \\
\hline 13 & Mirror Images & & $x$ & & \\
\hline 14 & Clocks & & $x$ & & \\
\hline 15 & Plan Drawing & & $x$ & & \\
\hline 16 & Koh's Test & & $x$ & & \\
\hline 17 & Yerkes' Test & & $x$ & & \\
\hline 18 & Impressive Speech & & & & $x$ \\
\hline 19 & Attributive Genative & & & $x$ & \\
\hline 20 & Spatial Relations & & $x$ & $x$ & \\
\hline 21 & Comparative Construction & & & & \\
\hline 22 & Inverted Grammar & & $x$ & $x$ & \\
\hline 23 & Complex Grammar & & & $x$ & $x$ \\
\hline 24 & Memory for Objects & & $x$ & & \\
\hline 25 & Memory for Sentences & & & $x$ & $x$ \\
\hline 26 & Memory for Visual Aids & & $x$ & & $x$ \\
\hline 27 & Memory-Pictogram & & $x$ & & $x$ \\
\hline 28 & Picture Interpretation & $x$ & $x$ & & \\
\hline 29 & Picture Sequence & & $x$ & & $x$ \\
\hline 30 & Parable & & $x$ & $x$ & $x$ \\
\hline 31 & Definition & & & $x$ & $x$ \\
\hline 32 & Comparison & & $x$ & $x$ & \\
\hline 33 & Logical Relations & & $x$ & & $x$ \\
\hline 34 & Arithmetical Relations & & $x$ & & $x$ \\
\hline
\end{tabular}

* SA Selective Attention Factor; SIM Simultaneous Processing Factor;

V Verbal Reading Factor; SUCC Successive Processing Factor. 
Table 3: Product Moment Correlations Between the Clinical Interviews and the Factors of the Base Line Test. (For clarity only significant correlations are shown)

\begin{tabular}{|c|c|c|c|c|c|}
\hline \multirow[b]{2}{*}{ Test } & \multirow[b]{2}{*}{ Name of Test } & \multicolumn{4}{|c|}{ Factors of Baseline Test } \\
\hline & & $\begin{array}{l}\text { Selective } \\
\text { Attention }\end{array}$ & $\begin{array}{l}\text { Simul- } \\
\text { taneous }\end{array}$ & $\begin{array}{c}\text { Reading } \\
\text { Verbal }\end{array}$ & Successive \\
\hline 1 & Handedness & & & & \\
\hline 2 & Verbal Hemisphere & & & & $0.25(0.02)$ \\
\hline 3 & Motor Hand Function & $0.28(0.01)^{*}$ & $0.38(0.00)$ & & \\
\hline 4 & Rhythmic Structures & $0.28(0.01)$ & & $0.27(0.02)$ & $0.33(0.00)$ \\
\hline 5 & Visual Functions & & & $0.31(0.01)$ & \\
\hline 6 & Reading Fluency & & & $0.44(0.00)$ & $0.30(0.00)$ \\
\hline 7 & Simple Pictures & & $0.26(0.02)$ & & \\
\hline 8 & Complex Pictures & & & & \\
\hline 9 & Silhouette Photo & & & $0.25(0.02)$ & \\
\hline 10 & Overlapping Pictures & & $0.34(0.00)$ & & \\
\hline 11 & Chessboard Figures & & $0.27(0.02)$ & & \\
\hline 12 & Patterns & & $0.43(0.00)$ & & \\
\hline 13 & Mirror Images & & $0.31(0.01)$ & & \\
\hline 14 & Clocks & & $0.31(0.01)$ & & \\
\hline 15 & Plan Drawing & & & $0.28(0.01)$ & \\
\hline 16 & Koh's Test & & $0.56(0.00)$ & & \\
\hline 17 & Yerkes' Test & & $0.28(0.12)$ & & \\
\hline 18 & Impressive Speech & & & & \\
\hline 19 & Attributive Genative & & & & $0.31(0.01)$ \\
\hline 20 & Spatial Relations & & $0.28(0.01)$ & $0.24(0.03)$ & \\
\hline 21 & Comparative construction & & $0.37(0.00)$ & & \\
\hline 22 & Inverted Grammar & & $0.31(0.00)$ & $0.22(0.04)$ & $0.30(0.01)$ \\
\hline 23 & Complex Grammar & & $0.26(0.02)$ & & \\
\hline 24 & Memory for Objects & & $0.28(0.01)$ & $0.35(0.00)$ & \\
\hline 25 & Memory for Sentences & & & & $0.41(0.00)$ \\
\hline 26 & Memory for Visual Aids & & $0.35(0.00)$ & & \\
\hline 27 & Memory-Pictogram & & $0.43(0.00)$ & & $0.28(0.01)$ \\
\hline 28 & Picture Interpretation & & & & \\
\hline 29 & Picture Sequence & & $0.29(0.01)$ & & $0.35(0.00)$ \\
\hline 30 & Parable & & $0.41(0.00)$ & $0.22(0.04)$ & $0.23(0.03)$ \\
\hline 31 & Definition & & & & $0.24(0.03)$ \\
\hline 32 & Comparison & & $0.22(0.04)$ & & \\
\hline 33 & Logical Relations & & $0.34(0.00)$ & & $0.34(0.00)$ \\
\hline 34 & Arithmetical Relations & & $0.26(0.02)$ & & \\
\hline
\end{tabular}

* Significance levels are shown in brackets

The protocol for these tests followed that of Luria and the test items themselves were in most cases identical. In a few instances an adaptation was made allowing for the age of the children and the differences in culture. The test kit from which the stimulus material was drawn did not include a dichotic listening tape. A special tape was prepared for this purpose by splicing together lengths of audio tape. This tape was used to 
determine hemisphericity. A detailed description of the tests is not possible in the context of this paper and the reader is referred to Christensen, 1974.

\section{Results and discussions}

The table of correlations provides strong evidence that the psychometric model is a valid representation of Luria's neurological model. With only a few exceptions the individual clinical tests used by Luria showed significant positive correlations with the predicted factors of the base line study. The simultaneous factor showed significant and relatively high positive correlations with a high proportion of the clinical tasks. This reflects the fact that many of these tasks are spatial in nature, such as reading a clock or comparing patterns. In addition to the obvious correlations between the simultaneous factor and the spatial tests there are some correlations which may surprise those who are not familiar with Luria's writings. Thus, item 21, Comparative Construction, required the subject to respond to a task which appears to be verbal and/or successive. "Kate is fairer than Margaret, but darker than Jane. Who is the fairest?" Luria has demonstrated that patients with damage to the area of the brain responsible for simultaneous processing, the occipito-parietal region have difficulty with this type of problem in addition to difficulty with spatial relations (Luria, 1966).

The successive factor showed positive and significant correlations with most of the predicted items in the battery of clinical tasks, the one exception being Motor Hand Function. In this task the children were required to place their hands in a variety of positions following the examiner's movements. The table of correlations shows that selective attention and simultaneous processing ability were much more significant determinants of this skill than was successive ability.

The reading/verbal factor showed positive correlations with most of the expected tasks and with a number of tasks which had not been predicted Rhythmic Structures (ie. following a tapping pattern, silhouette photo and plan drawing) It seems likely, that the children used their verbal skill as a strategy for solving what might be regarded as nonverbal problems.

Finally, it is noted that the Selective Attention factor only reached a significant level of correlation with two of the tasks. This result may reflect the fact that the selective attention factor was defined mainly through tests which also loaded significantly on the successive factor. In the present selection of clinical tasks most of the tests are more closely related to simultaneous processing. This paper has briefly outlined a model of information processing based on Luria's simultaneous/successive theory. Evidence has been presented which demonstrates the validity of the 
psychometric model in terms of Luria's clinical tasks. The model has much to offer the educational technologist since it suggests the possibility of adapting instruction to suit the needs of the individual learner in relatively simple ways. The clinical studies which have been undertaken highlight an important feature of information processing the distinction between mode of presentation and mode of processing. It cannot be assumed that the mode of presentation will necessarily be the mode of processing. Verbal statements may require simultaneous processing and some visuals may be more easily processed verbally.

The identification of learners who are high in simultaneous processing and low in successive processing and those who are low in simultaneous processing and high in successive processing suggests the need to develop specific teaching strategies for these groups. The educational technologist has available the tools necessary for individualising learning. Specifically, the videodisc, linked to the microcomputer allows individuals to follow alternative pathways in attaining learning objectives. The Luria model provides a sound theoretical framework for the development of suitable alternative programs.

\section{Bibliography}

Cronbach, L.J. and Snow, R.E. Aptitudes and Instructional Methods. New York: Irvington Press, 1977.

Christensen, A.L. Luria's Neurological Investigation. Text, Manual and Test Cards. New York: Spectrum, 1974.

Das, J.P. Structure of cognitive abilities: evidence for simultaneous and successive processing. Journal of Educational Psychology, 1973, 65(1), 103108.

Fitzgerald, D. Behavioural research in education. University of New England 1973.

Green, K.N. An examination of a model of individual differences in sequential and simultaneous processing for the study of aptitude treatment interaction. PhD Thesis, University of New England 1977.

Kirby, J.R. and Das, J.P. Information Processing and Human Abilities. Journal of Experimental Psychology, 1978, 70(1) 58-66.

Luria, A.R. Human brain and psychological processes. New York: Harper and Row, 1966.

Ransley, W.K. The development of a psychometric model of information processing in young children based on Luria's theory of brain functioning. PhD Thesis, University of New England 1981.

Salomon, G. Heuristic models for the generation of aptitude treatment interaction hypotheses. Review of Educational Research, 1972, 42(3) 327343. 
Salomon, G. \& Cohen, A.A. Television formats, mastery of mental skills and the acquisition of knowledge. Journal of Educational Psychology, 1977, 69(5) 612-619.

Salomon, G. Media and symbol systems as related to cognition and learning. Journal of Educational Psychology, 1979, 71(2) 131-148.

Walton, J.E. Sequential and simultaneous information processing abilities and their interaction with instructional treatments in senior high school mathematics. PhD Thesis, University of New England 1983.

Please cite as: Angus, J. (1985). The Luria model of information processing. Australian Journal of Educational Technology, 1(1), 59-67.

http: / / www.ascilite.org.au/ajet/ajet1/angus.html 